\title{
Intra-abdominal robot-assisted vasovasostomy of obstructive azoospermia in an Asian population following multiple bilateral inguinal herniorrhaphy in childhood: a case report and literature review
}

\author{
Tao Wang ${ }^{1,2}$, Zhe Yu ${ }^{1,2}$, Zhuo Liu ${ }^{1,2}$, Xiaming Liu ${ }^{1,2}$, Mingchao Li $^{1,2}$, Longjie Gu ${ }^{1,2}$, Ruzhu Lan ${ }^{1,2}$, \\ Shaogang Wang ${ }^{1,2}$, Jun Yang ${ }^{1,2}$, Jihong Liu ${ }^{1,2}$ \\ ${ }^{1}$ Department of Urology, Tongji Hospital, Tongji Medical College, Huazhong University of Science and Technology, Wuhan, China; ${ }^{2}$ Institute of \\ Urology, Tongji Hospital, Tongji Medical College, Huazhong University of Science and Technology, Wuhan, China \\ Correspondence to: Jihong Liu. 1095 Jiefang Avenue, Wuhan 430030, Hubei, China. Email: jhliu@tjh.tjmu.edu.cn.
}

\begin{abstract}
Iatrogenic injury to the vas deferens is an indication for vasovasostomy (VV). Various surgical approaches, including pure microsurgical VV (MVV), pelviscrotal laparoscopic-assisted VV (LAVV), and intra-abdominal robot-assisted VV (RAVV), have been reported to restore vasal patency. MVV is often faced a formidable challenge to provide tension-free $\mathrm{VV}$ due to an inadequate vas deferens length. Alternatively, pelviscrotal LAVV is much more effective for the identification and retrieval of the pelvic vas deferens prior to performing MVV. However, vasal laparoscopic mobilization could still be limited by insufficient vasal length for extracorporeal transfer in some cases. The addition of robotic assistance, on the other hand, allows the performance of "in-situ" vasal anastomoses and offers unique features compared with pure MVV/LAVV. However, few such approaches have been described in the literature. This study presents the initial results and validation of robot-assisted VV in an Asian population who had undergone triple herniorrhaphy. Briefly, Intra-operative findings demonstrated a large defect of the vas deferens, and a two-layer bilateral tension-free RAVV was performed to pursue the possibility of naturally achieved pregnancy. With our promising results, intra-abdominal RAVV may be described as a practical approach for cases with iatrogenic large defects of the vas deferens within the inguinal canal.
\end{abstract}

Keywords: Azoospermia; vasovasostomy (VV); laparoscopy; robotics; case report

Submitted Feb 10, 2021. Accepted for publication May 05, 2021.

doi: $10.21037 /$ tau-21-133

View this article at: http://dx.doi.org/10.21037/tau-21-133

\section{Introduction}

Common causes of obstructive azoospermia (OA) include vasectomy, congenital anomalies, infection and iatrogenic injuries (1). Inguinal vasal obstruction (IVO), specifically, occurs following hernia repairs in childhood and is potentially unrecognized in real-life scenarios (2). The incidence of injury was found to be as high as $9 \%$ in subfertile patients with a history of childhood herniorrhaphy (3). The mechanism of IVO can be cutting, crushing, overstretching or cauterization injury.

The latest guidelines suggest that couples in which the male partner is diagnosed with IVO undergo testicular exploration sperm extraction (TESE)/microsurgical or percutaneous epididymal sperm aspiration (MESA or PESA) with intracytoplasmic sperm injection (ICSI) to achieve pregnancy (4). However, some couples still prefer to achieve pregnancy naturally, especially since the announcement by the Chinese government that the iconic one-child policy would finally been replaced by a universal two-child policy (5).

Unfortunately, surgical vasovasostomy (VV) of IVO is a clinical challenge. The obstruction is commonly inguinal or retroperitoneal, and the vas deferens can be encompassed in 


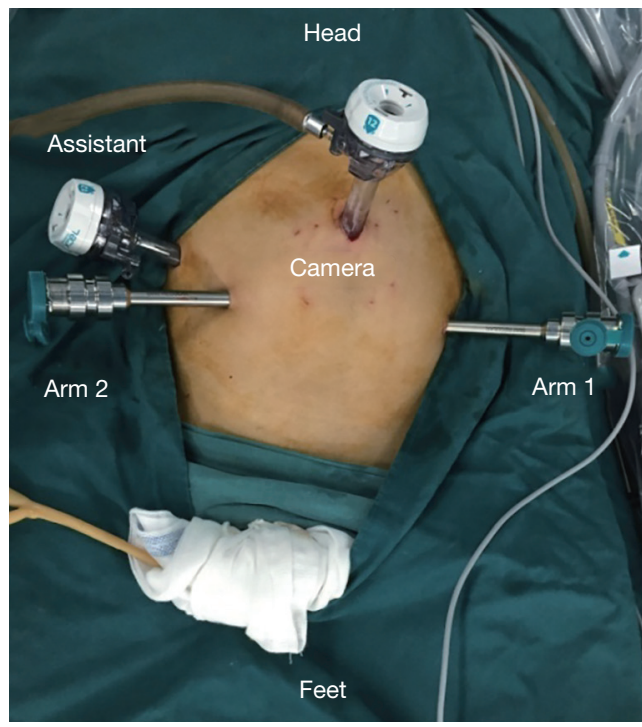

Figure 1 Port placement for the daVinci SI surgical system. The triangle-distribution includes two robotic working ports for the black diamond microforceps (Arm 1 and Arm 2), and one umbilical port for the zero-degree robotic camera. Another assistant port is located next to the right robotic working ports.

surrounding fibrotic tissues, thus making identification and reconstruction with pure microsurgical VV (MVV) almost impossible. Despite an alternative approach, pelviscrotal laparoscopic-assisted VV (LAVV), has been introduced to identify and retrieve additional length of the vas deferens, which would then be anastomosed to the scrotal vas deferens extracorporeally for standard MVV. The overall patency rate still represents a relevant gap with a very negative return for the patients $(6,7)$ because LAVV could still be limited by tension or an unsatisfactory reconstructive angle.

Recent studies have demonstrated the feasibility of inguinal robotic-assisted VV (RAVV) and that it may offer some potential advantages $(8,9)$. The addition of robotic assistance, on the other hand, allows the performance of "in-situ" vasal anastomoses and offers unique features compared with pure MVV and LAVV. However, the application of robotics has not been increased. According to the current literature, we are the first Asian center to report a successful intra-abdominal RAVV in patient with IVO after multiple herniorrhaphy using the da Vinci robotic system. Additionally, we summarize the pros and cons of the techniques described previously in the literature.

We present the following article in accordance with the CARE reporting checklist (available at http://dx.doi. org/10.21037/tau-21-133).

\section{Case presentation}

A 22-year-old man who had undergone triple bilateral herniorrhaphy (at the age ranging from 1 to 9 years) was referred to our institution. Multiple semen analyses revealed azoospermia. Diagnosis of suspected IVO was performed as described in detail elsewhere (10). All procedures performed in studies involving human participants were in accordance with the ethical standards of the institutional and/or national research committee(s) and with the Helsinki Declaration (as revised in 2013). Written informed consent was obtained from the patient for publication of this case report and any accompanying images.

Bilateral intra-abdominal RAVV was performed on April 6, 2016. After receiving general anesthesia, the patient was placed in a supine position. Intuitive Surgical (Sunnyvale, CA, USA) offers a four-arm da Vinci type Si robotic system. Two black diamond microforceps were loaded in the left and right arms. However, the third arm, usually loaded with micro-Potts scissors, was idle in the present case. The zerodegree camera port and 2 robot arm ports were distributed as triangles (Figure 1).

To identify the vas deferens, the peritoneum was opened at the level of the internal inguinal ring and extensive scarring was noted bilaterally with the vas deferens. The narrow segment of the vas deferens was approximately $2.8 \mathrm{~cm}$ (left) and $3.3 \mathrm{~cm}$ (right) and encompassed in surrounding fibrotic tissues. The available vas deferens was insufficient to transfer extracorporeally for MVV/LAVV, and intra-abdominal RAVV was an optimal choice.

Excision of the vasal obstructed segment was performed bilaterally (Figure 2). Then, the epididymis tail was squeezed to push vasal fluid. Vasal fluid was collected by using a rubber sheet, which could be delivered through an assistance port and transferred to a slide. In the present case, motile sperm were noted in vasal fluid under microscopic examination, therefore, the possibility of ipsilateral epididymal obstruction was otherwise negative. The distal vasal segments were intubated and irrigated with methylene blue after $3 \mathrm{Fr}$ ureteral catheter placement into the vasal lumen. Given the presence of a change in urine color, the patency of the distal vasal segment was confirmed.

A 1-cm inguinal incision was made, and the proximal vas deferens was bluntly mobilized by fingers. By doing so, we obtained an extra length of the vas deferens in a very short time, and the two ends of the vas deferens were able to re- 


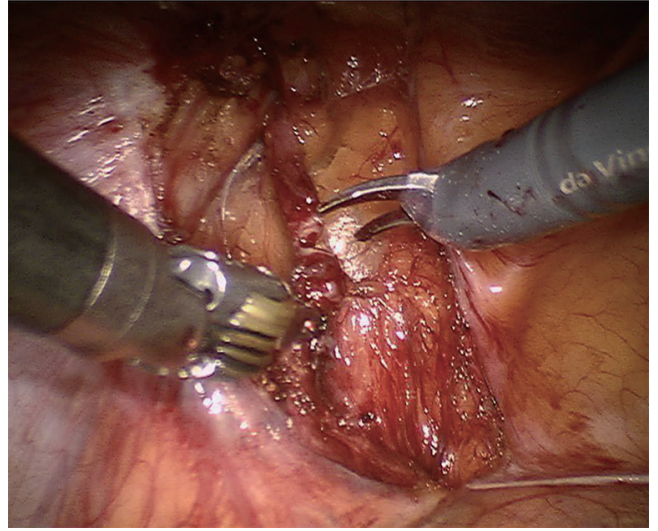

Figure 2 Intraoperative images demonstrate the initial identification of narrow segments of the vas deferens.

approximate. Compared to full-time robotic mobilization of the pelvic vas, it is a time-saving technique.

A two-layer VV was performed similarly to that used in vasectomy reversal. Briefly, end-to-end VV was achieved by mucosal anastomosis using six double-armed $10-0$ nylon sutures (Figure $3 A, B$ ), and seromuscular anastomosis was completed using six 8-0 nylon sutures (Figure 3C). Six final 7-0 sutures were placed for vasal adventitia closure (Figure $3 D$ ). The contralateral vas deferens was similarly reanastomosed.

\section{Results}

The total operative time was 207 minutes, and there were no intraoperative complications. A semen analysis performed 3 months postoperatively demonstrated a sperm concentration of $35.4 \times 10^{6} \mathrm{~mL}^{-1}$ and $18.18 \%$ progressive motility. Twins (two boys) were born on August 6, 2018.

\section{Discussion}

More than 20 million inguinal herniorrhaphy procedures have been performed worldwide (11). However, the possibility of male infertility as a consequence of herniorrhaphy is not well established. IVO is caused mainly by inadvertent resection of part of the vas deferens during previous herniorrhaphy. Delayed IVO may result from extrinsic compression or blood supply injury. The detection of unilateral IVO is not easy because these cases are usually nonazoospermic. However, bilateral IVO can result in azoospermia that may need to be emphasized in individuals who are currently under fertility investigations.
In cases of bilateral IVO, vasal reconstruction can be used to achieve natural fertility, whereas the alternative procedure of ICSI is a means of artificial reproduction with a relevant burden to the female partner. Associated risks, such as ovarian hyperstimulation, multiple pregnancy, and complications of the procedures for oocyte retrieval, may affect the female partner twice as often if a second child is planned. Thus, some couples pursue vasal reconstruction, despite the long operation time and uncertain results.

Since injuries occur in early childhood, a relatively long obstructive interval increases the likelihood of secondary epididymal obstruction. Evaluation of vasal fluid from the proximal end of the vas deferens, therefore, is the first step in making decisions about the type of surgical repair. Once microscopic examination of vasal fluid reveals sperm, an ipsilateral or crossed VV is performed.

Various surgical approaches, including pure MVV, pelviscrotal LAVV, and intra-abdominal RAVV, have been reported to restore vasal patency. Generally, pure MVV at the inguinal region after inguinal herniorrhaphy is extremely difficult and time consuming. According to the literature, the overall patency rate of pure MVV varies from approximately $56.5-76.7 \%$ (12-14), which is lower than those in reports of microsurgical vasectomy reversal. Technical problems are caused mainly by severe fibrosis, scarring, and adhesion that develop after herniorrhaphy and microsurgical anastomosis in the deep inguinal region. Abnormal anatomy and ischemic atrophy may result in negative detection of vasal ends. The distal end of the vas deferens was found at the internal inguinal ring or in the pelvic cavity in $57 \%$ of cases, and more than $3 \mathrm{~cm}$ of the vas deferens had been resected in $37 \%$ of cases $(15,16)$. Thus, opening the peritoneum is sometimes helpful to find the distal vas deferens. However, even if the remnants of the vas deferens are found, MVV is often faced with a formidable challenge to provide tension-free $\mathrm{VV}$ due to an inadequate length of the vas deferens. This component may have been devitalized or too separated, making approximation difficult and causing the anastomosis to be under tension, not to mention that it holds the risk of jeopardizing the previous herniorrhaphy. These findings highlighted the significant limitation of pure MVV in this challenging cohort, despite pure MVV is much less expensive than the other two procedures.

Alternatively, pelviscrotal LAVV is much more effective for the identification and retrieval of the pelvic vas deferens prior to performing pure MVV. This technique allows the pelvic vas deferens to be mobilized laparoscopically, 

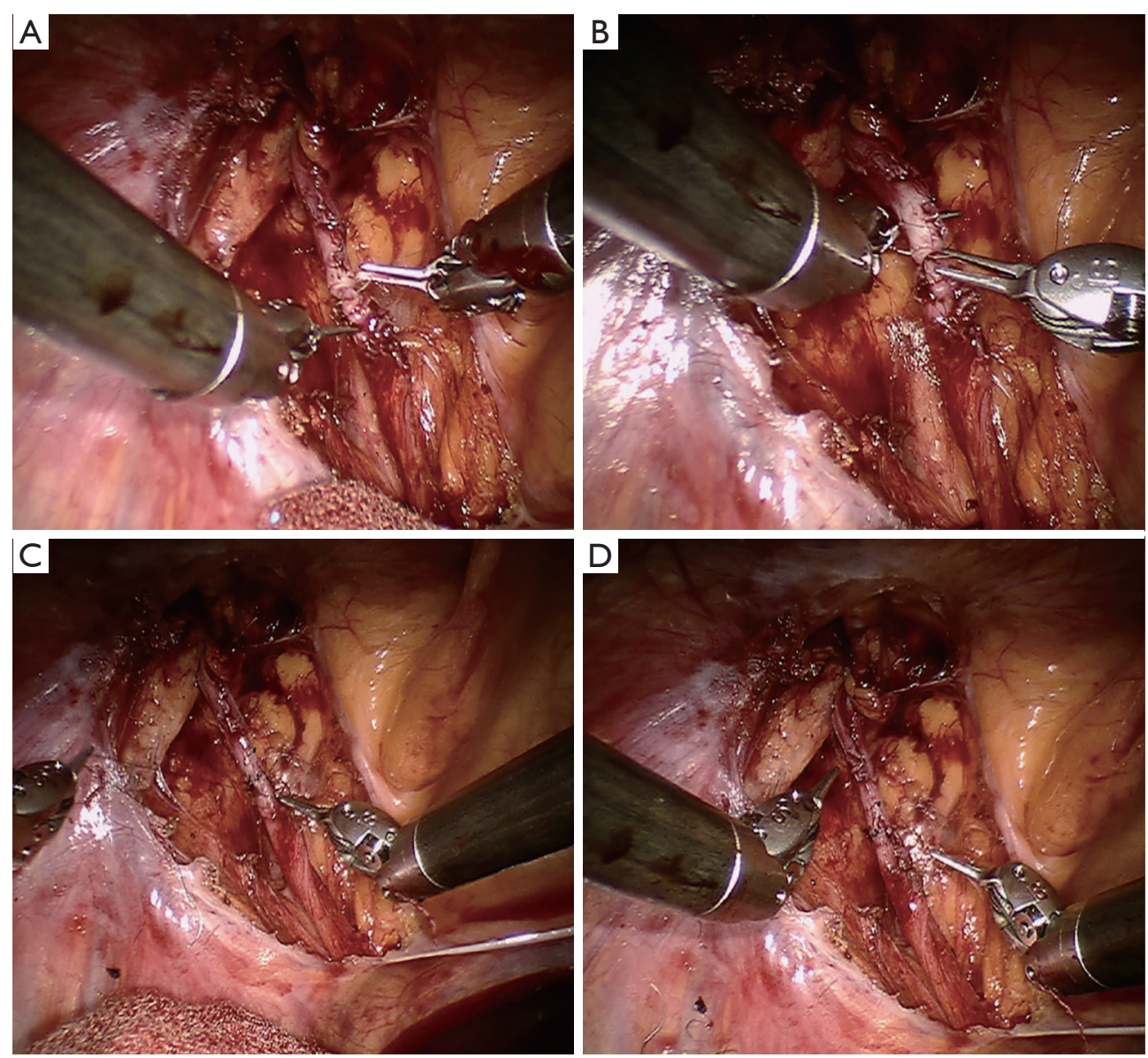

Figure 3 Intraoperative image demonstrates anterior mucosa stitches, anterior seromuscular stitches and the finished product. (A,B) Anterior 10-0 stitches placed. (C), Anterior seromuscular stitches. (D) Completed intra-abdominal vasovasostomy anastomosis.

detached from the internal ring, pulled out of the pelvis through the external inguinal ring, and then anastomosed to the scrotal vas deferens, enabling the inguinal vas deferens to be bypassed in a minimally invasive fashion to achieve additional length for the extracorporeal transfer of the vas deferens $(6,7)$. Approximately $5.83 \pm 0.65 \mathrm{~cm}$ of the vas deferens could be mobilized using this technique (17), and there is no risk of hernia recurrence. Outcomes of a series of 25 patients demonstrated that the overall patency rate was $68 \%$ (18). However, vasal laparoscopic mobilization could still be limited by extensive fibrosis at the inguinal internal ring or insufficient intra-abdominal vasal length for extracoporeal transfer, especially in obese patients. Even if the distal vas deferens is successfully transferred, VV could still be limited by tension or an unsatisfactory reconstructive angle. In addition, pelviscrotal LAVV needs to be coupled with MVV. MVV itself is technically difficult, and achieving good outcomes requires rigorous microsurgical training (19).

Robotic assistance offers unique features with standard laparoscopic surgery. In contrast to laparoscopic techniques, RAVV does not need to extracorporeally transfer the vas segment. The ability to handle the 10-0 sutures by black diamond microforceps ensures "in-situ" vasal reconstruction. Theoretically, the "in-situ" reconstruction technique results in an adequate vasal length for tensionfree anastomosis. Additionally, "in-situ" reconstruction gives a second chance for the crossover procedure in selected patients.

This procedure presents several unique challenges. The length of retroperitoneal dissection of the vas deferens is an initial problem. In cases with large defects of the vas deferens, more of the proximal vas deferens must be separated. On the other hand, an overlong vas deferens increases vasal oscillation. Thus, we suggest 
isolating the optimal length of the vas deferens, to avoid overmobilization.

Another technical difficulty is caused by the great discrepancy in the vasal diameter between the proximal and distal ends. In vasectomy reversal, the proximal vas deferens does not distend much when a sperm granuloma is present. In patients with IVO due to childhood inguinal herniorrhaphy, on the other hand, no sperm granuloma is present because sperm is not produced until much later in life. This may be responsible for the large differences in vasal diameter. Therefore, a microsurgical two-layer technique is required to avoid sperm leakage. With the introduction of the MVV, a two-layer vasal anastomosis has become the gold standard. Although the incidence of patency for the modified 1-layer technique is similar to that after a two-layer procedure with a meta-IR of 1.04 (95\% CI, 1.00-1.08) among vasectomy reversal procedures (20), the two-layer technique shows a better patency rate than the single-layer method among 133 post-herniorrhaphy patients (15).

The third arm of the robot can be loaded with micro-Potts scissors, but some authors believe this adds unnecessarily to the cost (21). In the present case, we used two black diamond microforceps to tear additional nylon sutures on knots. Thus, the total out-of-pocket cost was reduced. The da Vinci robot is associated with substantial costs in terms of acquisition, annual maintenance and disposable costs. However, cost could be solved by wider dissemination of the technology.

In general, there appears to be increased operative efficiency with robotic platforms compared with microsurgical platforms. In cases of vasectomy reversal, the use of robotic assistance may decrease operative duration and improve the rate of recovery of postoperative total motile sperm counts (22). The literature suggests a learning curve associated with robotic microsurgery, despite the surgeon's previous extensive background in microsurgical and robotic surgery $(23,24)$. Intra-abdominal RAVV maintains the advantages of the laparoscopic approach, including complete elimination of tremors, minimal invasiveness, high-definition three-dimensional visualization, high effectiveness in identifying distal vasal ends, bypassing the inguinal segment and eliminating the risk of disrupted prior hernia repair. However, further research and the results of large-scale supplementation trials would provide data to elucidate the use of intra-abdominal RAVV in comparison to MVV. Overall, robotic systems may be the most promising new technique for inguinal VV.

To our knowledge, two groups have previously reported the feasibility of intra-abdominal RAVV $(8,9)$. Currently, no group has reported the management of extensive abnormal anatomy in the inguinal region resulting from multiple herniorrhaphy at childhood by intra-abdominal RAVV. These data further demonstrated that both patients with susceptible multiple vasal injuries may elect to proceed with intra-abdominal RAVV. Many couples prefer natural pregnancy. However, it is difficult to estimate the obstructive site and length of the vas deferens preoperatively. If sufficient vasal length is present to transfer the vas segment extracorporeally, MVV/LAVV may be performed. Otherwise, intra-abdominal RAVV is the optimal choice. Effective communication is suggested to avoid any communication pitfalls.

Given the limited nature of the current study, additional studies are required in larger patient series. As with any technology, further evaluation and longer follow-up are needed to determine the feasibility and cost-effectiveness of intra-abdominal RAVV.

\section{Conclusions}

Inguinal herniorrhaphy is the most common cause of iatrogenic IVO. However, conventional microsurgical reconstruction to restore fertility is extremely difficult when IVO has occurred because of direct injuries and significant fibrotic reactions to previous herniorrhaphy. Although laparoscopic techniques provide some advantages over pure MVV, pelviscrotal LAVV is not feasible in cases of inadequate intra-abdominal vasal length. Intraabdominal RAVV on the other hand enables "in-situ" VV, revolutionizing techniques to perform $\mathrm{VV}$ in traditionally challenging locations while maintaining the advantages of the laparoscopic approach.

\section{Acknowledgments}

Funding: None.

\section{Footnote}

Reporting Checklist: The authors have completed the CARE reporting checklist. Available at http://dx.doi.org/10.21037/ tau-21-133

Conflicts of Interest: All authors have completed the ICMJE uniform disclosure form (available at http://dx.doi. org/10.21037/tau-21-133). The authors have no conflicts of 
interest to declare.

Ethical Statement: The authors are accountable for all aspects of the work in ensuring that questions related to the accuracy or integrity of any part of the work are appropriately investigated and resolved. All procedures performed in studies involving human participants were in accordance with the ethical standards of the institutional and/or national research committee(s) and with the Helsinki Declaration (as revised in 2013). Written informed consent was obtained from the patient for publication of this Case report and any accompanying images.

Open Access Statement: This is an Open Access article distributed in accordance with the Creative Commons Attribution-NonCommercial-NoDerivs 4.0 International License (CC BY-NC-ND 4.0), which permits the noncommercial replication and distribution of the article with the strict proviso that no changes or edits are made and the original work is properly cited (including links to both the formal publication through the relevant DOI and the license). See: https://creativecommons.org/licenses/by-nc-nd/4.0/.

\section{References}

1. Chan PT, Brandell RA, Goldstein M. Prospective analysis of outcomes after microsurgical intussusception vasoepididymostomy. BJU Int 2005;96:598-601.

2. Sheynkin YR, Hendin BN, Schlegel PN, et al. Microsurgical repair of iatrogenic injury to the vas deferens. J Urol 1998;159:139-41.

3. Taniguchi H, Iwamoto T, Ichikawa T, et al. Contemporary outcomes of seminal tract re-anastomoses for obstructive azoospermia: A nationwide Japanese survey. Int J Urol 2015;22:213-8.

4. Jungwirth A, Diemer T, Kopa Z, et al. EAU Guidelines on Male Infertility. Available online: https://uroweb.org/ guideline/male-infertility

5. Zeng $\mathrm{Y}$, Hesketh T. The effects of China's universal twochild policy. Lancet 2016;388:1930-8.

6. Shaeer OK, Shaeer KZ. Laparoscopy-assisted pelvi-scrotal vasovasostomy. Andrologia 2004;36:311-4.

7. Kim A, Shin D, Martin TV, et al. Laparoscopic mobilization of the retroperitoneal vas deferens for microscopic inguinal vasovasostomy. J Urol 2004;172:1948-9.

8. Barazani Y, Kaouk J, Sabanegh ES Jr. Robotic intraabdominal vasectomy reversal: A new approach to a difficult problem. Can Urol Assoc J 2014;8:E439-41.

9. Trost L, Parekattil S, Wang J, et al. Intracorporeal RobotAssisted Microsurgical Vasovasostomy for the Treatment of Bilateral Vasal Obstruction Occurring Following Bilateral Inguinal Hernia Repairs with Mesh Placement. J Urol 2014;191:1120-5.

10. Wang J, Liu Q, Zhang YP, et al. Treatment for vas deferens obstruction following childhood herniorrhaphy. Urology 2018;112:80-4.

11. Fusco P, Cofini V, Petrucci E, et al. Unilateral paravertebral block compared with subarachnoid anesthesia for the management of postoperative pain syndrome after inguinal herniorrhaphy: a randomized controlled clinical trial. Pain 2016;157:1105-13.

12. Shin D, Lipshultz LI, Goldstein M, et al. Herniorrhaphy with polypropylene mesh causing inguinal vasal obstruction: a preventable cause of obstructive azoospermia. Ann Surg 2005;241:553-8.

13. Pasqualotto FF, Pasqualotto EB, Agarwal A, et al. Results of microsurgical anastomosis in men with seminal tract obstruction due to inguinal herniorrhaphy. Rev Hosp Clín Fac Med Sao Paulo 2003;58:305-9.

14. Chen XF, Wang HX, Liu YD, et al. Clinical features and therapeutic strategies of obstructive azoospermia in patients treated by bilateral inguinal hernia repair in childhood. Asian J Androl 2014;16:745-8.

15. Matsuda T. Diagnosis and treatment of post-herniorrhaphy vas deferens obstruction. Int J Urol 2000;7:S35-8.

16. Matsuda T, Muguruma K, Hiura Y, et al. Seminal tract obstruction caused by childhood inguinal herniorrhaphy: results of microsurgical reanastomosis. J Urol 1998; 159:837-40.

17. Buch JP, Woods T. Retroperitoneal mobilization of the vas deferens in the complex vasovasostomy. Fertil Steril 1990;54:931-3.

18. Shaeer OK, Shaeer KZ. Pelviscrotal vasovasostomy: refining and troubleshooting. J Urol 2005;174:1935-7.

19. Belker AM, Thomas AJ Jr, Fuchs EF, et al. Results of 1,469 Microsurgical Vasectomy Reversals by the Vasovasostomy Study Group. J Urol 1991;145:505-11.

20. Herrel LA, Goodman M, Goldstein M, et al. Outcomes of microsurgical vaovasostomy for vasectomy reversal: a metaanalysis and systematic review. Urology 2015;85:819-25.

21. Fleming C. Robot-assisted vasovasostomy. Urol Clin North Am 2004;31:769-72.

22. Parekattil SJ, Brahmbhatt JV. Robotic approaches for male infertility and chronic orchialgia microsurgery. Curr Opin Urol 2011;21:493-9. 
23. Kavoussi PK, Harlan C, Kavoussi KM, et al. Robotassisted microsurgical vasovasostomy: the learning curve for a pure microsurgeon. J Robot Surg 2019;13:501-4.

24. Parekattil SJ, Gudeloglu A, Brahmbhatt J, et al. Robotic

Cite this article as: Wang T, Yu Z, Liu Z, Liu X, Li M, Gu L, Lan R, Wang S, Yang J, Liu J. Intra-abdominal robotassisted vasovasostomy of obstructive azoospermia in an Asian population following multiple bilateral inguinal herniorrhaphy in childhood: a case report and literature review. Transl Androl Urol 2021;10(6):2521-2527. doi: 10.21037/tau-21-133
Assisted Versus Pure Microsurgical Vasectomy Reversal: Technique and Prospective Database Control Trial. J

Reconstr Microsurg 2012;28:435-44. 